\title{
Surgical Outcomes of Orbital Fracture Reconstruction Using Patient-Specific Implants
}

Blumer, Michael ; Essig, Harald ; Steigmiller, Klaus ; Wagner, Maximilian Eberhard ; Gander, Thomas

\begin{abstract}
Purpose Patient-specific implants (PSIs) are known to yield reliable outcomes in orbital wall fracture reconstruction (high precision, smoother operating techniques, and shorter surgical duration). This study analyzed the surgical error and clinical and esthetic outcomes of orbital reconstructions with PSIs. Methods This ambispective cohort study enrolled patients who underwent orbital reconstruction using PSIs between October 2016 and January 2018. The study endpoints were surgical error, indication and duration of surgery, long-term sequelae, revision surgeries, and surgical complications. Surgical error was analyzed by superimposing the postoperative implant position onto the preoperative virtual plan. Both qualitative (heat map) and quantitative (distance) measurements were obtained. Results Three patients were enrolled prospectively and 23 were enrolled retrospectively. Indications for surgery were defect size (25 patients), diplopia (10 patients), impaired eye motility (4 patients), and significant enophthalmos (6 patients). At the last patient visit, there were 5 cases of diplopia, 1 case of exophthalmos, and 6 cases of slight enophthalmos of incremental degree. In terms of surgical error, a mean distance of $0.6 \mathrm{~mm}$ (95\% CI 0.49-0.76), with mean maximal distance of $3.4 \mathrm{~mm}$ (95\% CI 2.79-4.02), was noted. No revision surgery was necessary. Lid malposition complications were not observed. However, 1 case each of symblepharon and scleral show were observed. No time-saving component was observed. Conclusion PSI use in orbital reconstruction guarantees a preplanned 3D anatomical shape with a mean surgical error of just $0.6 \mathrm{~mm}$. Our clinical results were similar to those of other protocols; however, warranting a complex 3D anatomical shape also in large orbital fractures with a low mean surgical error is feasible by using PSI.
\end{abstract}

DOI: https://doi.org/10.1016/j.joms.2020.12.029

Posted at the Zurich Open Repository and Archive, University of Zurich

ZORA URL: https://doi.org/10.5167/uzh-196234

Journal Article

Accepted Version

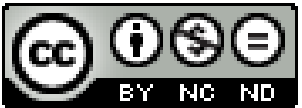

The following work is licensed under a Creative Commons: Attribution-NonCommercial-NoDerivatives 4.0 International (CC BY-NC-ND 4.0) License.

Originally published at:

Blumer, Michael; Essig, Harald; Steigmiller, Klaus; Wagner, Maximilian Eberhard; Gander, Thomas (2021). Surgical Outcomes of Orbital Fracture Reconstruction Using Patient-Specific Implants. Journal of Oral and Maxillofacial Surgery, 79(6):1302-1312.

DOI: https://doi.org/10.1016/j.joms.2020.12.029 


\section{Surgical Outcomes of Orbital Fracture Reconstruction Using Patient- Specific Implants}

Michael Blumer, MD, DMD ${ }^{1,2}$, Harald Essig, MD, DMD ${ }^{1,2}$, Klaus Steigmiller, B Sc ${ }^{3}$, Maximilian Eberhard Wagner, MD, DMD, ${ }^{2}$, Thomas Gander, MD, DMD, ${ }^{1,2}$

${ }^{1}$ These authors contributed equally to this work

${ }^{2}$ Consultant, Department Oral \& Maxillofacial Surgery, University Hospital, Zurich, Switzerland

${ }^{3}$ Researcher, Department of Biostatistics and Epidemiology, Biostatistics and Prevention Institute, University of Zurich, Zurich, Switzerland

\section{Corresponding author:}

Michael Blumer, MD, DMD,

UniversitätsSpital Zürich

Klinik für Mund-, Kiefer- und Gesichtschirurgie

Frauenklinikstrasse 24

8091 Zürich

Telephone number: +41432539575

Email: Michael.Blumer@usz.ch

Keywords:

Orbital fractures, computer-assisted surgery, virtual reconstruction, computer-aided design, customized implants, patient specific implants 


\begin{abstract}
$\underline{\text { Purpose }}$

Patient-specific implants (PSIs) are known to yield reliable outcomes in orbital wall fracture reconstruction (high precision, smoother operating techniques, and shorter surgical duration). This study analyzed the surgical error and clinical and aesthetic outcomes of orbital reconstructions with PSIs.
\end{abstract}

Methods:

This ambispective cohort study enrolled patients who underwent orbital reconstruction using PSIs between October 2016 and January 2018. The study endpoints were surgical error, indication and duration of surgery, long-term sequelae, revision surgeries, and surgical complications. Surgical error was analyzed by superimposing the postoperative implant position onto the preoperative virtual plan. Both qualitative (heat map) and quantitative (distance) measurements were obtained.

$\underline{\text { Results: }}$

Three patients were enrolled prospectively and 23 were enrolled retrospectively. Indications for surgery were defect size (25 patients), diplopia (10 patients), impaired eye motility (4 patients), and significant enophthalmos (6 patients). At the last patient visit, there were 5 cases of diplopia, one case of exophthalmos, and six cases of slight enophthalmos of incremental degree. In terms of surgical error, a mean distance of $0.6 \mathrm{~mm}$ (95\% CI $0.49-0.76)$, with mean maximal distance of $3.4 \mathrm{~mm}(95 \%$ CI 2.79-4.02), was noted. No revision surgery was necessary. Lid malposition complications were not observed. However, one case each of symblepharon and scleral show were observed. No time-saving component was observed.

\title{
Conclusion:
}

PSI use in orbital reconstruction guarantees a preplanned 3D anatomical shape with a mean surgical error of just $0.6 \mathrm{~mm}$. Our clinical results were similar to those of other protocols; however, warranting a complex 3D anatomical shape also in large orbital fractures with a low mean surgical error is feasible by using PSI. 


\section{Introduction}

Patient-specific implants (PSI) are becoming popular among surgeons treating complex orbital fractures. Due to their location, these common midface fractures can be a challenge to operate on, owing to their limited view and maneuvering space. Sequelae such as diplopia, ocular motility disturbances, and enophthalmos may arise with or without surgery, with optical neuropathy or blindness being possible complications of these fractures.

Reconstruction of orbital fractures may be performed through a mirroring technique that virtually reconstructs a healthy orbit $[1,2]$. The PSIs are designed using the adapted mirrored orbit and produced using additive titanium manufacturing techniques [3-6].

The purpose of this study was to analyze specific PSI features, as they are considered to facilitate the surgical procedure by reducing the operating time and offering a smoother operating technique. Further, the rigidity of PSIs may be a favorable characteristic, especially in cases of large defects or a missing posterior ledge.

The investigators wanted to introduce a new method of 3D quality measurements of PSIs and hypothesized that the mean surgical error in orbital reconstruction would be about $1 \mathrm{~mm}$. This would lead to low functional and aesthetic complications and shorter time spent in the operating room. Therefore, surgical error was measured, functional and aesthetic complications were assessed, and operating room time was calculated.

\section{Methods}

$\underline{\text { Study Design }}$

To address the research purpose of this study, an ambispective cohort design was developed. It was approved by the institutional review board Kantonale Ethikkommission Zürich (ref. no. 2016-00833), and Swissmedics (ref. no. 10000149). All the participants in this study were patients of the University Hospital Zurich; all participants signed an informed consent for further use of their clinical data and/or for the prospective analysis of this study (Fig. 1).

The study population included patients who experienced unilateral orbital fractures that were reconstructed with PSIs at the university hospital between October 2016 and January 2018. To be 
included in the study, patients had to sign a general consent form. Patients had to sign an additional specific declaration of consent for the prospective arm. They also had to be 18 years of age or older. The patients were excluded from the study if they were underage, if there was a significant comorbid disease that contraindicated surgery, if informed consent was missing or it was not possible to obtain informed consent, or if orbital reconstruction was performed without a PSI.

The clinic information system was searched for the clinical and radiological information. All data were imported into electronic Case Report Forms (eCRF) using a web-based software for clinical data capture (secuTrial ${ }^{\circledR}$, interActive Systems, Berlin, Deutschland). This study was monitored by the Department Clinical Trial Center of the University Hospital Zurich.

PSI Design and Planning

Each implant was virtually designed by a clinician of the Department of Oral \& Maxillofacial Surgery. The files in Standard Tessellation (STL) format were sent to a supplier of medical technology surgical solutions (KLS Martin Group, Tuttlingen, Germany) for the control, edit, and production (using a selective laser melting method) of the PSI. The manufactured implants were not yet sterile and had to be sterilized in the hospital.

\section{$\underline{\text { Surgical Fracture Repair with PSI }}$}

All patients were operated on by consultants from the Department of Oral \& Maxillofacial Surgery under general anesthesia, following the disinfection and sterilization protocol of the hospital. A transconjunctival retroseptal approach was used in all cases. The fractured walls were dissected, and all orbital tissue was reduced before insertion of the PSI. A forced duction test was performed before and after implant insertion for the evaluation of ocular motility. The implant was set in the best fit position and secured with one screw. For surgical quality control, postoperative computed tomography was performed on the day after surgery.

\section{$\underline{\text { Surgical Error and Outcome Analysis: }}$}

For the analysis of surgical error with PSI use, the reconstructed orbit was segmented using iPlan ENT software (Brainlab, Feldkirchen, Germany) and digitally prepared for superimposition in the Meshlab software (Institute of Information Science and Technologies or CNR-ISTI, Rome, Italy). The original 
STL file was imported to CloudCompare (EDF, Paris, France) as a reference, and the segmented postoperative STL file was imported as a specimen (Fig. 2).

CloudCompare (EDF, Paris, France) was used to calculate the surgical error by superimposing the postoperative implant position onto the preoperative virtual plan. In this way, both qualitative (heat map) and quantitative (distance) assessment of surgical error were feasible. For quantitative assessment, the software measured the distance of each corresponding cloud point as a positive value. The error between the surgical result and the virtual plan was measured as mean distance, standard deviation (SD), and minimal and maximal distances between the two specimens.

Clinical data were registered in the electronic data capture software secuTrial ${ }^{\circledR}$ (interActive Systems $\mathrm{GmbH}$, Berlin, Germany) until closure of the data bank (after complete acquisition of the data).

Timepoints for clinical evaluation were preoperative, during inpatient stay (1-4 days postoperative), and during the outpatient phase (last follow-up: 3 months postoperative).

The primary endpoints of the study were surgical error, long-term sequelae, and duration of surgery. Surgical error was analyzed as mentioned above. The duration of surgery was analyzed in cases in which only the orbit was reconstructed during surgery, and this information was extracted from the operating report. Long-term sequelae, defined as binocular diplopia, enophthalmos, and exophthalmos, were assessed at the above mentioned timepoints. Naugle ophthalmometry was used to detect globe malposition.

Following were the secondary endpoints: surgical indications, associated ophthalmological injuries, surgical complications, and revision surgeries. The clinical information system was searched for revision surgeries and surgical complications (i.e. alteration in lid position, symblepharon, and infection). Surgical indications that were assessed included defect size, diplopia, ocular motility disturbances, and globe malposition. In addition, reports from the ophthalmologists were searched for associated ophthalmological injuries.

\section{$\underline{\text { Statistical analysis }}$}

For descriptive analysis, mean and SD or median and interquartile range (IQR) were calculated for continuous variables; number and percentage were calculated for categorical variables. Plots were used for a better description of the distributions and relationships. For all inferential estimates, results are 
reported as $95 \%$ Wald confidence intervals (CI) in case of approximately normal continuous outcomes, 95\% Wilson CIs for binary outcomes, and simultaneous multinomial 95\% CIs developed by Glaze and Sison [7] for multinomial variables. No subgroup or sensitivity analyses were performed due to the relatively small sample size; adjustments for confounding factors were also not made. Methods for dealing with missing data were not required since the data were complete. All analyses were conducted using R version 3.6.2 for Windows (R Foundation, Vienna, Austria) [8].

\section{Results}

In this study, 3 patients were enrolled prospectively and 23 patients were identified retrospectively. In the prospective group, one patient was excluded after enrollment as they wished for an early operation (Fig. 1). Fifteen patients were male (61.5\%), and the mean age was 47.8 years. Fall from a height less than $3 \mathrm{~m}$ and interpersonal violence were the main causes of fracture.

A sole orbital floor fracture (one wall) was diagnosed in 19 patients, while multiwall orbital fracture was observed in seven. Indications for surgical reconstruction were the defect size in 25 patients. In one patient, the zygomaticomaxillary complex fracture was operated on first due to significant facial asymmetry, followed by secondary reconstruction of the orbit due to emerging enophthalmos. Other indications for surgery included diplopia, ocular motility disturbances, and enophthalmos, present in 10 (38.5\%, $95 \%$ Wilson CI from $20.9 \%$ to $59.3 \%$ ), four (15.4\%, $95 \%$ Wilson CI from $5.0 \%$ to $35.7 \%$ ), and six patients $(23.1 \%, 95 \%$ Wilson CI from $9.8 \%$ to $44.1 \%)$, respectively.

The associated orbital injuries are presented in Table 1. On the last follow-up, traumatic anisocoria and hypesthesia of the second branch of the trigeminal nerve persisted in one and eight patients, respectively. Binocular diplopia, enophthalmos, and exophthalmos were considered long-term sequelae and measured at three timepoints (Table 2). Enophthalmos and exophthalmos were preoperatively and postoperatively measured as being present or absent, respectively; however, in the last follow-up visit, a Naugle exophthalmometer was used to assign a numerical value $(\mathrm{mm})$ for the conditions. This protocol may be considered ideal, as the swelling during the first two timepoints may complicate a precise reading of the ophthalmometer. 
The estimated proportion of diplopia was $19.2 \%$ (95\% Wilson CI $8.5 \%-37.9 \%$ ). Results for both enophthalmos and exophthalmos are presented in Table 3.

The analysis of surgical error revealed a mean distance of $0.6 \mathrm{~mm}$ (95\% CI $0.49-0.76)$; mean value of the maximal distance was $3.4 \mathrm{~mm}(95 \%$ CI $2.79-4.02)$. Plots for the visualization of distribution of the variables are shown in Figure 3.

There was no requirement for surgical revisions in any of the patients (Table 4).

Complications of the surgery were assessed during the last follow-up. No eyelid alterations, such as entropion or ectropion, were observed (Wilson 95\% CI from $0 \%$ to $16 \%$ ). One case each of symblepharon and scleral show were observed (3.8 \%, Wilson $95 \%$ CI from $0.2 \%$ to $21.6 \%$ ).

No visual correlation between increasing surgical error (mean distance) and increasing PSI area was observed. At most, a slight tendency toward increasing surgical error with increasing PSI area was noted, although a wide scatter was noted on visualization (Fig. 3). No association was observed between mean distance and one-wall or multiwall fractures (Fig. 4).

There were 21 cases without additional fractures (i.e., nasal fractures); in these cases, median operation time was $110 \mathrm{~min}$ (IQR $80-130 \mathrm{~min}, 95 \%$ CI from 87.5 to $122.5 \mathrm{~min}$ ). There was a tendency toward shorter surgical duration in patients with one-wall orbital fracture (Table 5).

Table 6 shows the analysis of the PSI area compared with the number of fractured orbital walls and preoperative diplopia. PSI area was larger in the group with multiwall orbital fractures; there was no difference in PSI area for preoperative diplopia.

\section{Discussion}

With this new method of 3D quality measurement, the study investigators analyzed the mean surgical error associated with the use of PSIs and hypothesized that it would be about $1 \mathrm{~mm}$. Further, the occurrence of functional and aesthetic complications would be low, and the time needed in the operating room would be minimal. We observed a very low surgical error with a mean distance of $0.6 \mathrm{~mm}$ between the virtual and surgical reconstructions. Furthermore, good clinical and aesthetic outcomes were observed with few instances of postoperative diplopia, enophthalmos, and exophthalmos. No surgical 
revisions were required, and the surgeries were performed in a reliable manner; however, no time-saving component was observed.

The main causes of orbital fractures were falls from a height of $<3 \mathrm{~m}$ and interpersonal violence. Onewall-fractures were present in most patients, and defect size was the main indication for surgical repair. Hypesthesia was present during the last patient visit in $30.8 \%$ of patients, whereas other associated preoperative ophthalmological injuries, such as impaired eye motility, orbital swelling and hematoma, ocular contusion, and subconjunctival hemorrhage, subsided quickly after surgery.

Previous studies have shown that PSIs may be beneficial for several reasons: reconstructions are considered to be simpler and more accurate, especially in complex orbital fractures; the stiffness of the PSI prevents unwanted bending during insertion; preoperative planning may avoid interferences during surgery [3-5].

In this study, demographic analyses showed that the majority of the patients were male and that falls from a standing height of $<3 \mathrm{~m}$ and interpersonal violence were the main etiologies of the fractures. These findings correspond with the existing literature [9-12].

The most frequent fracture-associated findings were subconjunctival hemorrhage, monocle hematoma, and orbital swelling, as reported in earlier studies [12]. Ocular motility disturbances and binocular diplopia were observed to be less frequent than that in in other studies [11].

Direct postoperative hypesthesia of the second trigeminal branch and orbital swelling arose as a surgical consequence.

Meanwhile, long-term sequelae such as binocular diplopia and enophthalmos were resolved immediately after surgery. At the end of the follow-up period, diplopia was observed in only five cases (19.2\%). This finding was slightly higher than that in a previous retrospective study on customized stock implants [13] (binocular diplopia in $8.8 \%$ of cases), and slightly lower than that in a prospective multicenter study on PSIs [11]. This discrepancy may be explained by the longer follow-up period of the preceding studies. Postoperative binocular diplopia is described in $8-42 \%$ of cases in the literature $[14,15]$. Another explanation for this discrepancy may be associated with the use of more expensive PSIs in our clinic for orbital fractures with large defects, and comminution of the transition zone (also called the "internal orbital buttress") or the posterior ledge. These structures are considered vital for 
successful reconstruction [16]. PSIs may be advantageous in such cases due to their rigid characteristics compared with malleable stock implants that have been customized on bio-models.

Long-term sequela of enophthalmos was observed in six patients (23.1\%); three had a value $\geq 2 \mathrm{~mm}$ on the Naugle ophthalmometer. In two patients, preoperative enophthalmos was observed; the patient with 3-mm enophthalmos initially underwent open reduction and fixation of the zygomaticomaxillary complex, as previously mentioned. Nonetheless, the postoperative 3D imaging showed a symmetrical reconstruction, in which the transition zone and the posterior ledge were meticulously reconstructed. This fact indicates that these globe malpositions may be a consequence of fat atrophy [17, 18], as reconstructions are performed and controlled using the best surgical modalities. In cases where a correction is necessary, this may be performed by secondary implant augmentation [19]; this procedure was not required in our patients.

Further, with no cases of exophthalmos in any patient, and enophthalmos occurring in $23.1 \%$ of patients, it raises the question of whether a slight allowance for "overcorrection" should be given during the preoperative planning, instead of just aiming to restore the obtained mirrored orbital volume ("true-toorigin" concept). One also has to be aware of the natural minor asymmetry in patients when using this true-to-origin concept with mirroring. Thus, the question of which localization and to what extent this "overcorrection" should happen is an interesting problem that may hopefully be addressed in future prospective studies.

In previous studies, assessment of surgical error has been performed by measuring the absolute maximal distances in distinct planes and comparing the reconstructed angulations of the orbital floor or orbital volumes with the unaffected side. In our opinion, superimposition of the segmented postoperative image onto the virtual reconstruction reveals the surgical error better, both qualitatively (heat mapping) and quantitatively (actual values). Therefore, the comparison of our results with those of other studies is not possible.

Accordingly, for the surgical error analysis, the postoperative 3D image was segmented and superimposed onto the preoperative planned PSI. To the best of our knowledge, this is the first study in the literature to do so. The calculated mean distance was $0.6 \mathrm{~mm}$ (95\% CI 0.49-0.76). This low mean surgical error also indicates that the 3D anatomical reconstruction was conducted correctly. This implies 
that the critical structures of transition zone and posterior ledge were reconstructed correctly. Therefore, a mean error of $0.6 \mathrm{~mm}$ between the planned and surgically achieved results is very good and a proof of a reliable 3D anatomical orbital reconstruction.

The larger value of the mean maximal distance $(3.4 \mathrm{~mm}$; $95 \%$ CI $2.79-4.02)$ was due to bony artifacts in the segmented surgical results, which were impossible to clean before the measurement (Fig 5). Keeping this in mind, a mean distance of $0.6 \mathrm{~mm}$ appears even more impressive.

Other difficulties encountered in segmenting the postoperative surgical results are shown in Fig. 6. PSI thickness was shown to be $\sim 1.2 \mathrm{~mm}$ due to a radiological metal artifact (dependent on rendering settings); however, the exact thickness of the PSI amounted to $0.3 \mathrm{~mm}$. This radiological artifact may also lead to measuring inaccuracies.

Other reasons for inaccuracies and real surgical errors were due to rotations of the PSI, especially in the coronal plane, during the reconstruction of the posterior ledge and transition zone. This finding could be easily detected using heat maps. This might explain the clinically favorable results despite the inaccuracies in actual measurements (Fig. 5). An earlier study demonstrated an accurate positioning of PSIs without the requirement for intraoperative imaging [5]; however, the rotational inaccuracies in this study show that even lower surgical error may be achieved with the use of both intraoperative imaging and superimposition.

A tendency toward lower surgical error (mean distance) was observed in the group with one-wall fractures and smaller PSI area; however, the difference was not clinically relevant. Therefore, we assume that a low surgical error may be achieved while utilizing PSI, even in cases with larger defects and multiple wall fractures, such as those involving the posterior ledge and transition zone.

Although the relevance of the following fact is unknown, we would like to report that no revision was required in the inpatient or outpatient setting, even though five patients showed binocular diplopia and six patients presented with enophthalmos. We believe that this may be because the sequelae were not bothersome to the patients.

Surgical complications included only one symblepharon and one scleral show. No lid alterations such as ectropion or entropion were observed. The safety and reliability of the transconjunctival retroseptal approach used in this study coincides with previous reports [20,21]. 
The median operation time was 110 minutes overall (IQR 80-130). Surgeries for one-wall orbital fractures were shorter (median $92 \mathrm{~min}$; IQR 72.5-116.3) compared to those for multiwall fractures (median $130 \mathrm{~min}$; IQR 125-145). This coincides with a previous study on orbital reconstructions using customized stock implants through 3D bio-models [13]. Therefore, compared with the bio-model method, the use of PSIs did not decrease the mean surgery time; however, the preoperative digital planning time was increased. Due to higher costs, our clinic uses PSIs only in complicated orbital fractures, or in educational surgeries. In contrast, the bio-model method is employed in simple orbital fractures or in complex fractures that are handled by more experienced surgeons. Therefore, the results of time comparison between these two methods should be interpreted with caution. For relevant cost and time comparisons, a study with standardized orbital fracture reconstructions performed by the same surgeons would have to be conducted.

Analyses of the area and type of orbital fractures showed an association between increasing area and multiwall orbital fractures, which seems consistent as multiwall fractures are considered to be larger in area than one-wall fractures. A surprising result was observed in the analysis of preoperative diplopia, which revealed no correlation between increasing area and binocular diplopia. This suggests that fracture location may be a compelling factor in the occurrence of diplopia.

A limitation of this study is its ambispective design and the small sample size. The need to undersign several documents on informed consent from different departments and clinics while being admitted for trauma confused many patients and discouraged participation in the prospective arm of this study. However, these factors also facilitated the feasibility of conducting analyses on the surgical error of orbital reconstruction. Furthermore, direct comparisons with other studies are not possible, as no other study known to the authors involved the measurement of a mean surgical error by the superimposition of postoperative implant position onto the preoperative virtual plan. For direct comparisons to other methods, more studies with this protocol would be needed.

The use of PSIs in orbital reconstruction guarantees a preplanned 3D anatomical shape with a low surgical error of $0.6 \mathrm{~mm}$. Furthermore, the rigidity of PSI allows a thorough reconstruction of delicate orbital areas such as the posterior ledge and the transition zone. Our clinical results were similar to those obtained through other protocols. However, compared with other methods, no time-saving component 
was observed in this study. Future studies comparing different orbital reconstruction methods in a standardized manner should be conducted.

\section{References}

1. Gellrich NC, Schramm A, Hammer B, Rojas S, Cufi D, Lagrèze W, Schmelzeisen R:

Computer-assisted secondary reconstruction of unilateral posttraumatic orbital deformity. Plast Reconstr Surg 110:1417, 2002

2. Blumer M, Gander T, Kruse Gujer A, Seifert B, Rucker M, Lubbers HT: Influence of Mirrored Computed Tomograms on Decision-Making for Revising Surgically Treated Orbital Floor Fractures. J Oral Maxillofac Surg 73:1982.e1, 2015

3. Gander T, Essig H, Metzler P, Lindhorst D, Dubois L, Rucker M, Schumann P: Patient specific implants (PSI) in reconstruction of orbital floor and wall fractures. J Craniomaxillofac Surg 43:126, 2015

4. Rana M, Chui CH, Wagner M, Zimmerer R, Rana M, Gellrich NC: Increasing the accuracy of orbital reconstruction with selective laser-melted patient-specific implants combined with intraoperative navigation. J Oral Maxillofac Surg 73:1113, 2015

5. Rana M, Holtmann H, Rana M, Kanatas AN, Singh DD, Sproll CK, Kübler NR, Ipaktchi R, Hufendiek K, Gellrich NC: Primary orbital reconstruction with selective laser melted core patient-specific implants: overview of 100 patients. Br J Oral Maxillofac Surg $57: 782,2019$

6. Ruiters S, Mombaerts I: Applications of three-dimensional printing in orbital diseases and disorders. Curr Opin Ophthalmol 30:372, 2019 
7. Glaz J, Sison CP: Simultaneous confidence intervals for multinomial proportions. J Stat Plan Inference 82:251, 1999

8. Team RC: R: A Language and Environment for statistical computing. R Foundation for Statistical Computing, Vienna, Austria., 2019

9. Bartoli D, Fadda MT, Battisti A, Cassoni A, Pagnoni M, Riccardi E, Sanzi M, Valentini V: Retrospective analysis of 301 patients with orbital floor fracture. J

Craniomaxillofac Surg 43:244, 2015

10. Blumer M, Kumalic S, Gander T, Lanzer M, Rostetter C, Rucker M, Lubbers HT: Retrospective analysis of 471 surgically treated zygomaticomaxillary complex fractures. J Craniomaxillofac Surg 46:269, 2018

11. Zimmerer RM, Ellis E, Aniceto GS, Schramm A, Wagner MEH, Grant MP, Cornelius C-P, Strong EB, Rana M, Chye LT, Calle AR, Wilde F, Perez D, Tavassol F, Bittermann G, Mahoney NR, Alamillos MR, Bašić J, Dittmann J, Rasse M, Gellrich N-C: A prospective multicenter study to compare the precision of posttraumatic internal orbital reconstruction with standard preformed and individualized orbital implants. J Craniomaxillofac Surg $44: 1485,2016$

12. Blumer M, Rostetter C, Johner J-P, Ebner JJ, Wiedemeier D, Rücker M, Gander T: Associated Ophthalmic Injuries in Patients With Fractures of the Midface. J Craniomaxillofac Trauma Reconstr 13:168, 2020

13. Blumer M, Pejicic R, Gander T, Johner JP, Held U, Wagner ME: Customized Titanium Reconstruction of Orbital Fractures Using a Mirroring Technique for Virtual Reconstruction and 3D Model Printing. J Oral Maxillofac Surg, 2020 
14. Boyette JR, Pemberton JD, Bonilla-Velez J: Management of orbital fractures: challenges and solutions. Clinical Ophthalmology (Auckland, N.Z.) 9:2127, 2015

15. Gavin Clavero MA, Simón Sanz MV, Til AM, Jariod Ferrer ÚM: Factors Influencing Postsurgical Diplopia in Orbital Floor Fractures and Prevalence of Other Complications in a Series of Cases. J Oral Maxillofac Surg 76:1725, 2018

16. Kunz C, Audigé L, Cornelius C-P, Buitrago-Téllez CH, Rudderman R, Prein J: The Comprehensive AOCMF Classification System: Orbital Fractures - Level 3 Tutorial. Craniomaxillofac Trauma Reconstr 7:S092, 2014

17. Chen CT, Huang F, Chen YR: Management of posttraumatic enophthalmos. Chang Gung Med J 29:251, 2006

18. Cole P, Kaufman Y, Hollier L: Principles of facial trauma: orbital fracture management. J Craniofac Surg 20:101, 2009

19. Boyette JR, Pemberton JD, Bonilla-Velez J: Management of orbital fractures: challenges and solutions. Clin Ophthalmol 9:2127, 2015

20. Gander T, Rostetter C, Blumer M, Wagner M, Schumann P, Wiedemeier DB, Rucker M, Essig H: Use of a monopolar microneedle device in a transconjunctival, retroseptal approach. J Craniomaxillofac Surg 45:1934, 2017

21. Raschke G, Rieger U, Bader RD, Schaefer O, Guentsch A, Schultze-Mosgau S: Outcomes analysis of eyelid deformities using photograph-assisted standardized anthropometry in 311 patients after orbital fracture treatment. J Trauma Acute Care Surg $73: 1319,2012$ 
Table 1: Associated orbital injuries at the following timepoints: preoperative, postoperative in hospital, and outpatient settings.

\section{Associated Orbital Injuries}

Preoperative Postoperative Follow-Up

\begin{tabular}{|c|c|c|c|}
\hline \multirow{2}{*}{ Hypesthesia } & 7 & 15 & 8 \\
\hline & $26.9 \%$ & $57.7 \%$ & $30.8 \%$ \\
\hline \multirow{2}{*}{ Impaired Motility } & 4 & 1 & $\mathbf{0}$ \\
\hline & $15.4 \%$ & $3.8 \%$ & $0.0 \%$ \\
\hline \multirow{2}{*}{ Orbital Swelling } & 7 & 15 & $\mathbf{0}$ \\
\hline & $26.9 \%$ & $57.7 \%$ & $0.0 \%$ \\
\hline \multirow{2}{*}{ Lid Laceration } & 6 & $\mathbf{0}$ & $\mathbf{0}$ \\
\hline & $23.1 \%$ & $0.0 \%$ & $0.0 \%$ \\
\hline \multirow{2}{*}{ Subconjunctival Hemorrhage } & 12 & 1 & $\mathbf{0}$ \\
\hline & $46.2 \%$ & $3.8 \%$ & $0.0 \%$ \\
\hline \multirow{2}{*}{ Monocle Hematoma } & 10 & 9 & $\mathbf{0}$ \\
\hline & $38.5 \%$ & $34.6 \%$ & $0.0 \%$ \\
\hline \multirow[b]{2}{*}{ Ocular Contusion } & 4 & $\mathbf{0}$ & $\mathbf{0}$ \\
\hline & $15.4 \%$ & $0.0 \%$ & $0.0 \%$ \\
\hline \multirow[b]{2}{*}{ Iris Injury } & 1 & $\mathbf{0}$ & $\mathbf{0}$ \\
\hline & $3.8 \%$ & $0.0 \%$ & $0.0 \%$ \\
\hline \multirow{2}{*}{ Anisocoria } & 1 & 1 & 1 \\
\hline & $3.8 \%$ & $3.8 \%$ & $3.8 \%$ \\
\hline
\end{tabular}


Table 2: Long-term sequelae diplopia, enophthalmos and exophthalmos at different timepoints.

\section{Long-Term Sequelae}

\begin{tabular}{|c|c|c|c|c|}
\hline & $\begin{array}{c}\text { Pre- } \\
\text { operative }\end{array}$ & $\begin{array}{c}\text { Post- } \\
\text { operative }\end{array}$ & & Follow-Up \\
\hline \multirow{2}{*}{ Diplopia } & 10 & 9 & & 5 \\
\hline & $38.5 \%$ & $34.6 \%$ & & $19.2 \%$ \\
\hline \multirow{6}{*}{ Enophthalmos } & & & \multirow[b]{2}{*}{$1 \mathrm{~mm}$} & 3 \\
\hline & & & & $11.5 \%$ \\
\hline & 6 & 1 & \multirow{2}{*}{$2 \mathrm{~mm}$} & 2 \\
\hline & $23.1 \%$ & $3.8 \%$ & & $7.7 \%$ \\
\hline & & & \multirow[b]{2}{*}{$3 \mathrm{~mm}$} & 1 \\
\hline & & & & $3.8 \%$ \\
\hline \multirow{6}{*}{ Exophthalmos } & & & \multirow{2}{*}{$1 \mathrm{~mm}$} & 1 \\
\hline & & & & $3.8 \%$ \\
\hline & $\mathbf{0}$ & $\mathbf{0}$ & \multirow{2}{*}{$2 \mathrm{~mm}$} & $\mathbf{0}$ \\
\hline & $0.0 \%$ & $0.0 \%$ & & $0.0 \%$ \\
\hline & & & \multirow[b]{2}{*}{$3 \mathrm{~mm}$} & $\mathbf{0}$ \\
\hline & & & & $0.0 \%$ \\
\hline
\end{tabular}

Table 3: Estimated proportion for enophthalmos (left) and exophthalmos (right) with $95 \%$ confidence interval by Sison and Glaz at last follow-up. 


\begin{tabular}{|c|c|c|c|c|c|c|c|}
\hline Enophthalmos & Estimation & $\begin{array}{c}\text { Lower } \\
\text { CI }\end{array}$ & Upper CI & Exophthalmos & Estimation & Lower CI & $\begin{array}{c}\text { Upper } \\
\text { CI }\end{array}$ \\
\hline absent & 0.77 & 0.65 & 0.94 & absent & 0.96 & 0.92 & 1 \\
\hline $1 \mathrm{~mm}$ & 0.12 & 0.00 & 0.28 & $1 \mathrm{~mm}$ & 0.04 & 0.00 & 0.10 \\
\hline $2 \mathrm{~mm}$ & 0.08 & 0.00 & 0.24 & $2 \mathrm{~mm}$ & 0.00 & 0.00 & 0.06 \\
\hline $3 \mathrm{~mm}$ & 0.04 & 0.00 & 0.21 & $3 \mathrm{~mm}$ & 0.00 & 0.00 & 0.06 \\
\hline
\end{tabular}

CI: confidence interval 
Table 4: Analysis of surgical error via CloudCompare, and revision surgery.

\section{Surgical Error \&}

\section{Revision}

\begin{tabular}{|c|c|c|c|c|c|c|c|c|}
\hline & Mean & $\begin{array}{l}\text { Lower } \\
\text { Range }\end{array}$ & $\begin{array}{l}\text { Upper } \\
\text { Range }\end{array}$ & $\begin{array}{c}\text { Lower } \\
\text { CI }\end{array}$ & $\begin{array}{c}\text { Upper } \\
\text { CI }\end{array}$ & SD & $\begin{array}{l}\text { Revision } \\
\text { Inpatient }\end{array}$ & $\begin{array}{c}\text { Revision } \\
\text { Outpatient }\end{array}$ \\
\hline Mean Distance & & & & & & & & \\
\hline $\begin{array}{c}\text { CloudCompare } \\
\text { Software }\end{array}$ & 0.6 & 0.2 & 1.6 & 0.5 & 0.8 & 0.4 & 0 & 0 \\
\hline Standard & & & & & & & & \\
\hline $\begin{array}{l}\text { Deviation } \\
\text { CloudCompare }\end{array}$ & 0.5 & 0.2 & 1.6 & 0.4 & 0.6 & 0.3 & 0 & 0 \\
\hline Software & & & & & & & & \\
\hline Maximal & & & & & & & & \\
\hline $\begin{array}{l}\text { Distance } \\
\text { CloudCompare }\end{array}$ & 3.4 & 1.7 & 8.4 & 2.8 & 4.0 & 1.6 & 0 & 0 \\
\hline Software & & & & & & & & \\
\hline
\end{tabular}


Table 5: Surgery duration in minutes.

\begin{tabular}{cccc}
\hline & Overall & 1 Wall Orbital Fracture & Multiwall Orbital Fracture \\
\hline $\mathbf{N}$ & 21 & 16 & 5 \\
\hline Operation Time & 110 & 92.5 & 130 \\
\cline { 2 - 4 }$($ median [IQR]) & {$[80-130]$} & {$[72-116.25]$} & {$[125-145]$} \\
\hline
\end{tabular}

IQR: interquartile range 
Table 6: Analyses of the patient-specific implant area stratified for number of fractured orbital walls and preoperative diplopia; Median and IQR values for Figures 8 and 9.

\begin{tabular}{|c|c|c|c|c|c|}
\hline & \multirow{2}{*}{ Overall } & \multicolumn{2}{|c|}{ Type of Orbital Wall Fracture } & \multirow{2}{*}{$\begin{array}{c}\text { Preoperative } \\
\text { Diplopia }\end{array}$} & \multirow{2}{*}{ No Diplopia } \\
\hline & & 1 Wall & Multiwall & & \\
\hline $\mathbf{n}$ & 26 & 19 & 7 & 10 & 16 \\
\hline Area PSI & 674,3 & 599,7 & 891,1 & 599,6 & 700,1 \\
\hline $\begin{array}{c}\mathbf{m m}^{2} \\
\text { (median } \\
[\mathrm{IQR}])\end{array}$ & $\begin{array}{l}{[511.4-} \\
798.0]\end{array}$ & {$[505.6-721.3]$} & {$[760.3-1032.2]$} & [513.5-761.9] & $\begin{array}{l}{[520.7-} \\
818.7]\end{array}$ \\
\hline
\end{tabular}

PSI: patient-specific implant; IQR: interquartile range 


\section{Figure Legends}

Fig. 1: Schematic flow chart of sample size enrollment.

Fig. 2: Superimposition of orbital reconstruction with patient-specific implant (PSI) (segmented from postoperative computed tomography; yellow) on the virtually planned PSI (red) (A). A heat map shows qualitative surgical error (B). Postoperative radiological control in coronal (C), axial (D), and sagittal planes (E).

Fig. 3: Scatterplot analyzing surgical error (mean distance) and patient-specific area Glaze.

Fig. 4: Boxplot analyzing surgical error (mean distance) and number of fractured orbital walls.

Fig. 5: The picture shows a postoperative 3D image in the sagittal plane with a superimposed virtual patient-specific implant (PSI) (A) and a magnified view on the right side (B). The implanted PSI seems to be $1.2 \mathrm{~mm}$ thick due to metal artifacts, whereas its true thickness is $0.3 \mathrm{~mm}$.

Fig. 6: Patient-specific implant (PSI), which is rotated in the coronal plane. The transition zone and posterior ledge are sufficiently reconstructed. A small bony artifact (red circle) increases the inaccuracy of the measurement further. View from the side (A) and above (B). 
Fig 1:

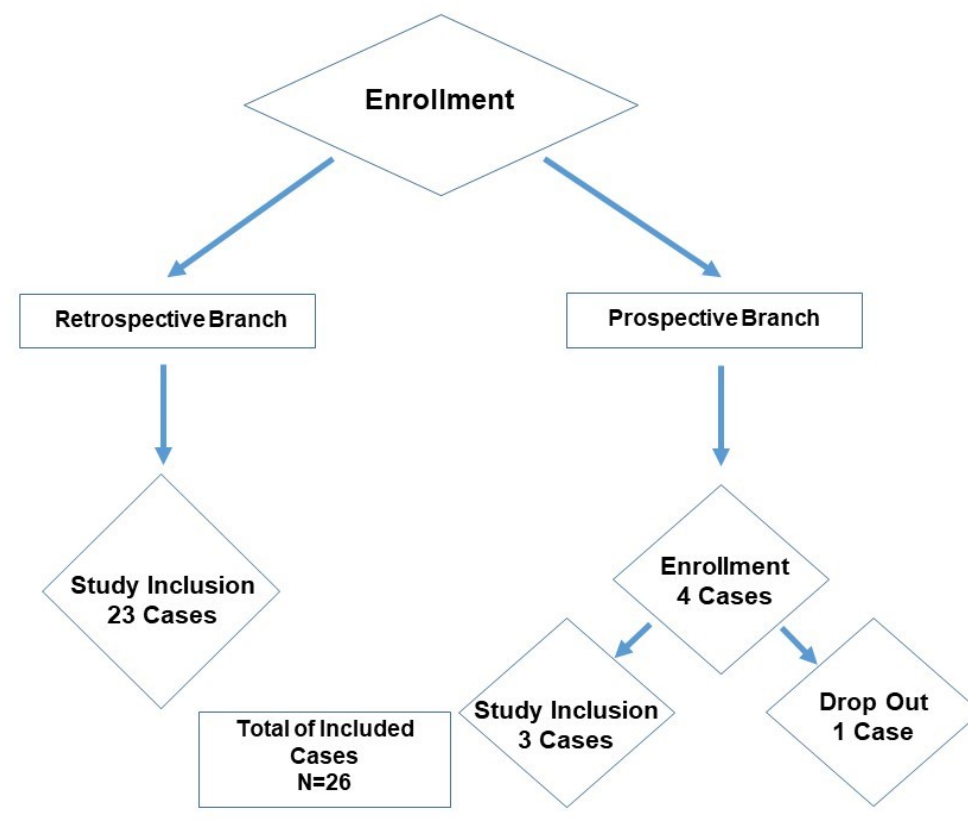

Fig 2A:

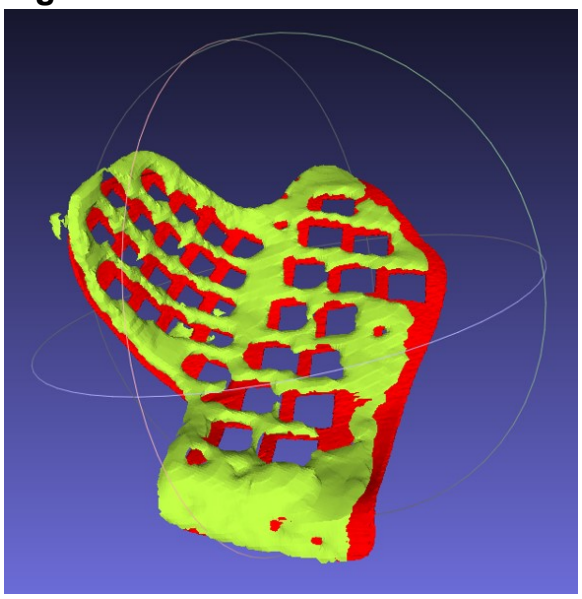

Fig 2B:

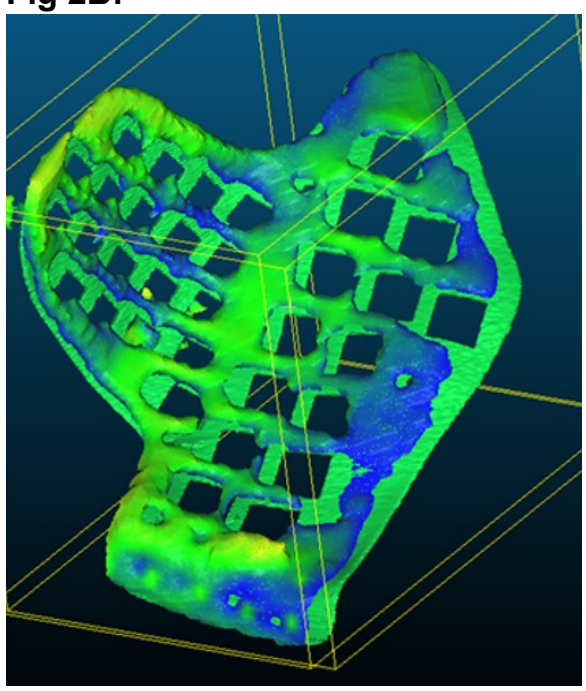


Fig 2C:

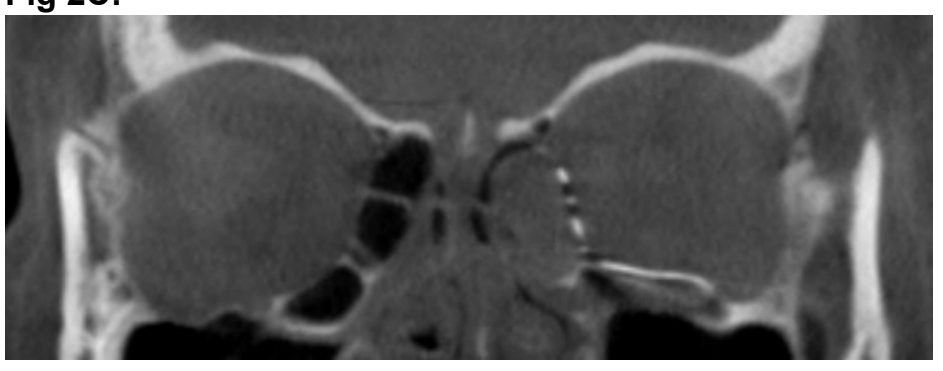

Fig 2D:

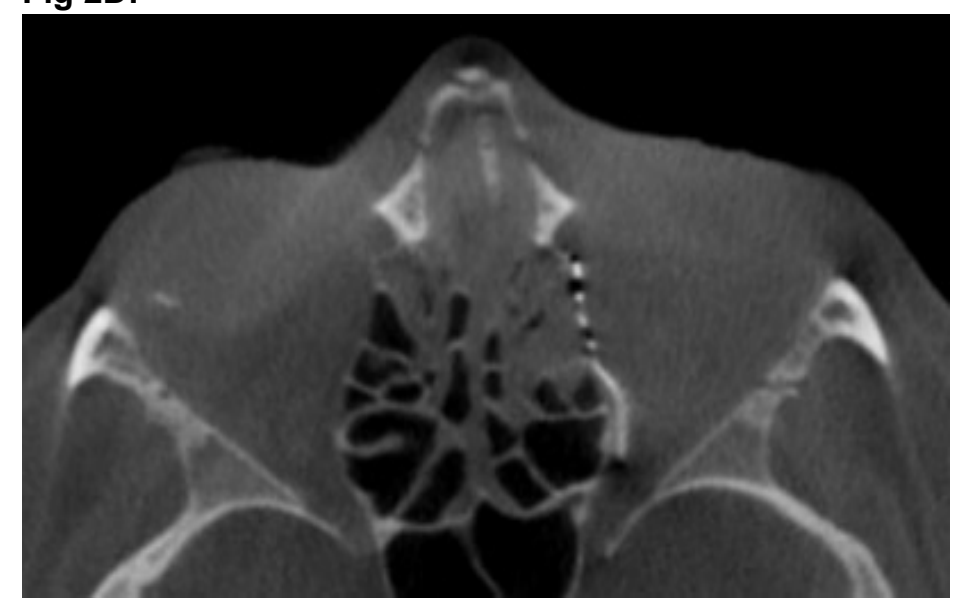

Fig 2E:

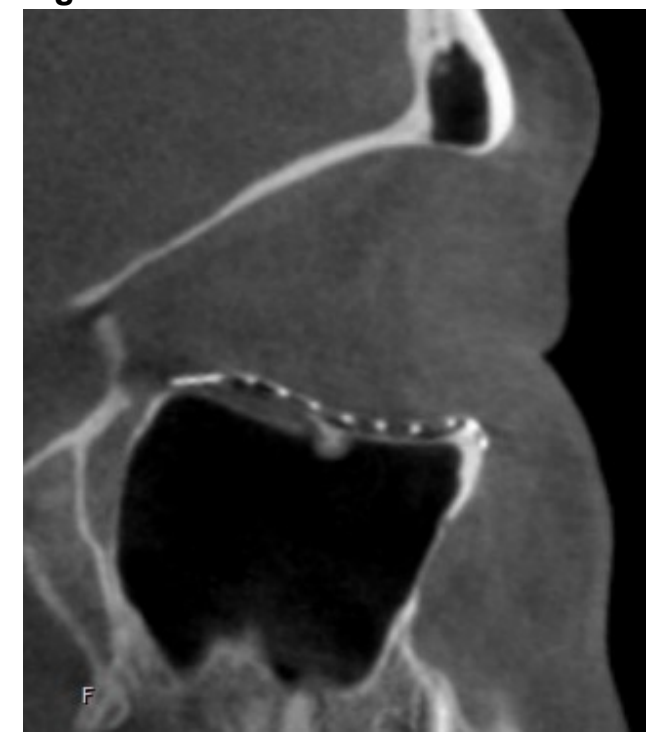


Fig 3:

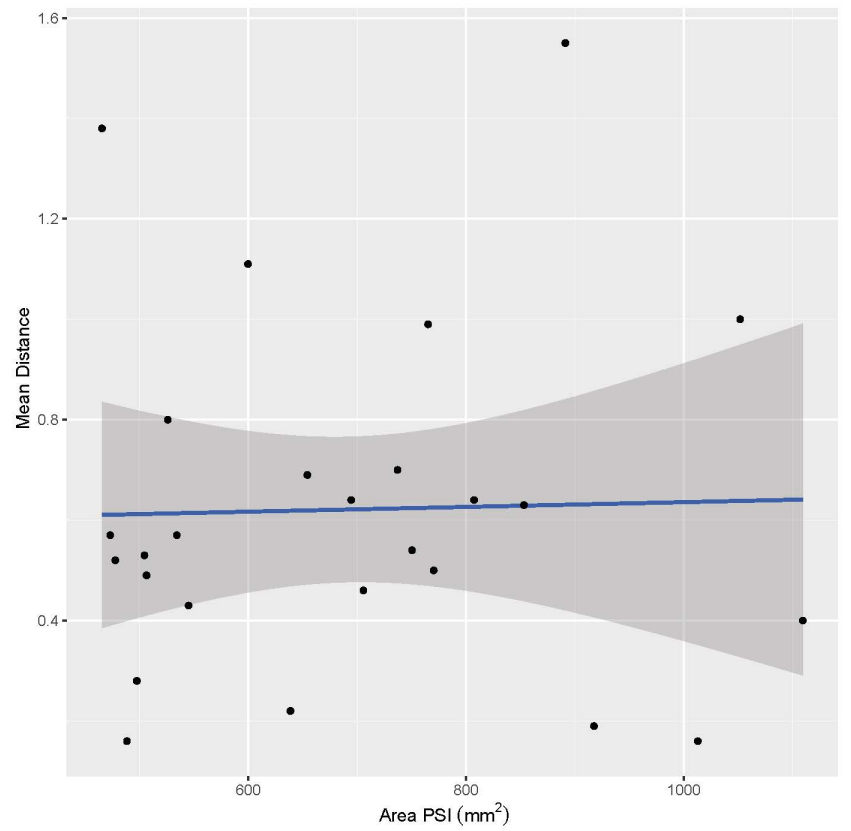

Fig 4:

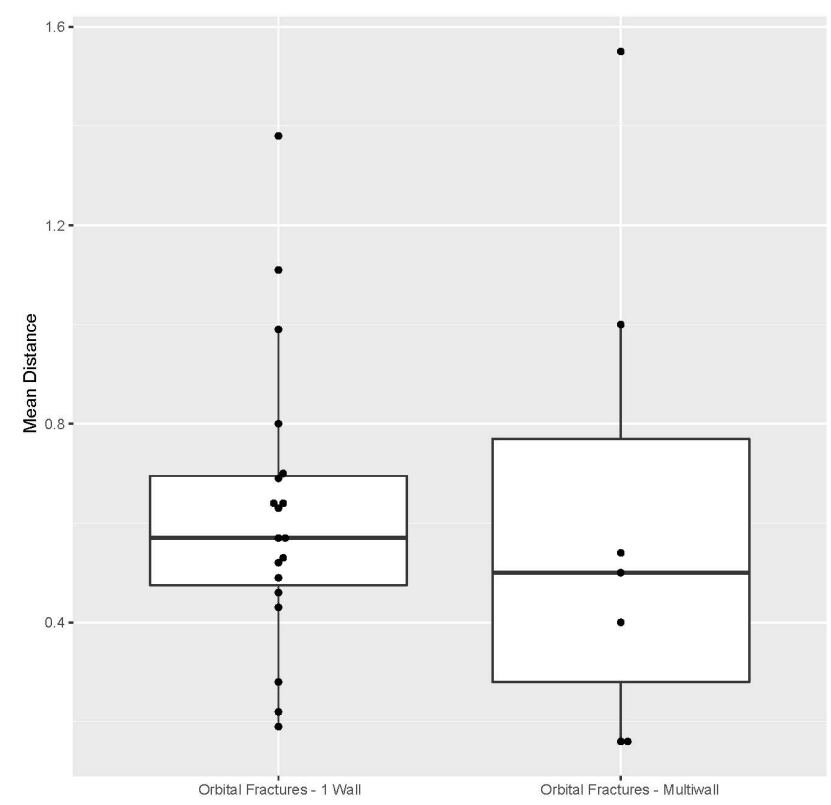


Fig 5A:

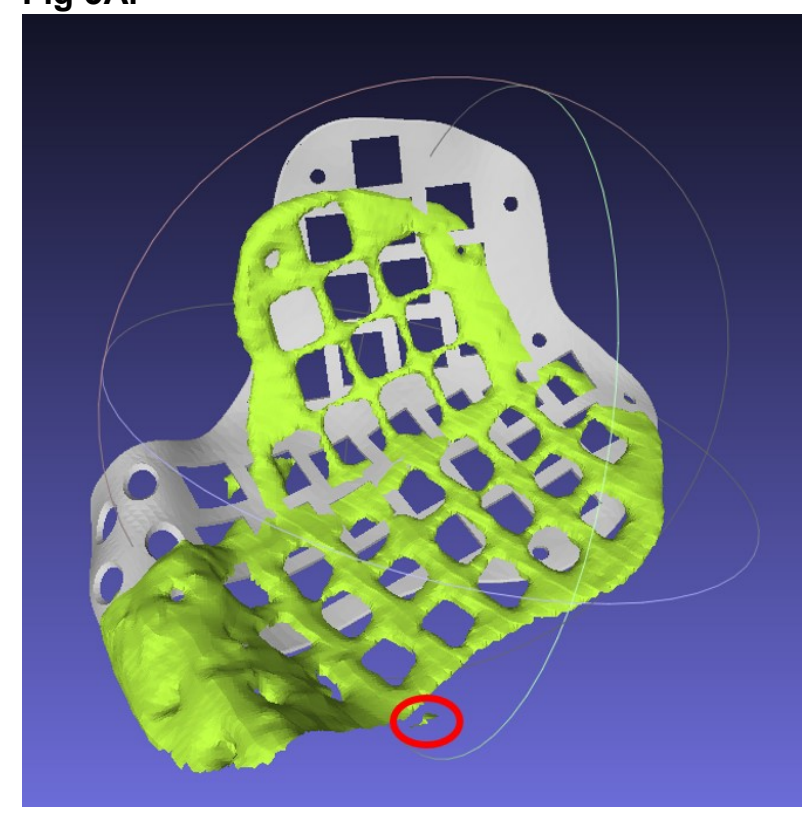

Fig 5B:

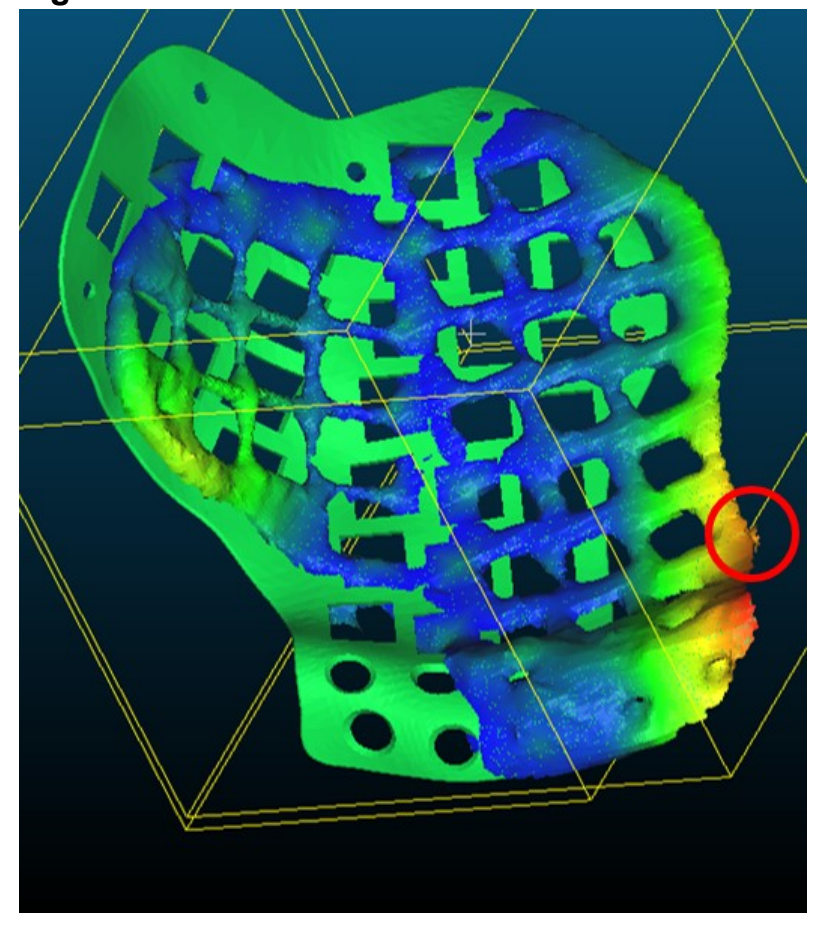


Fig 6A:

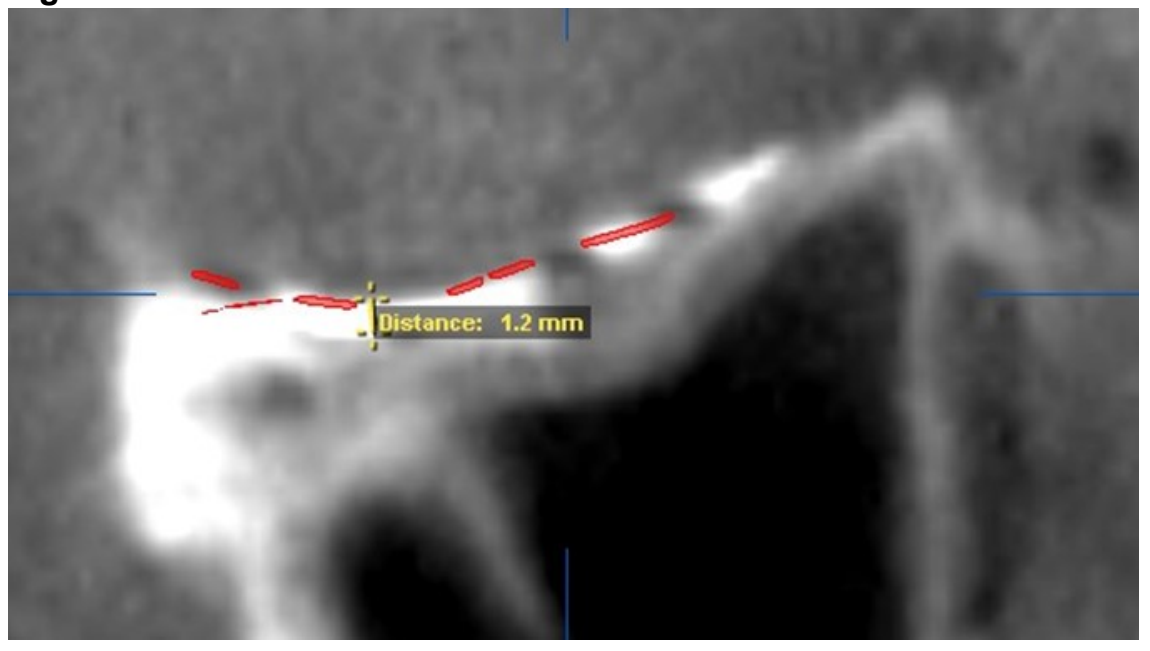

Fig 6B:

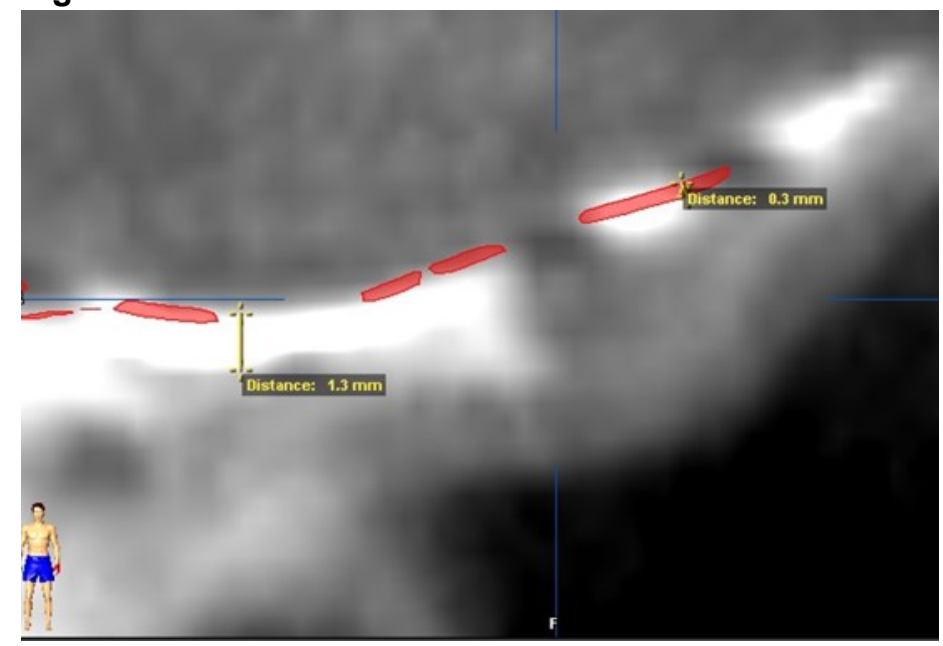

